\section{Research Square}

Preprints are preliminary reports that have not undergone peer review.

They should not be considered conclusive, used to inform clinical practice, or referenced by the media as validated information.

\title{
Anti-rhinovirus and anti-influenza virus activities of mucoactive secretolytic agents and plant extracts - a comparative in vitro study
}

\author{
Christin Walther \\ Jena University Hospital \\ Michaela Schmidtke ( $\square$ michaela.schmidtke@med.uni-jena.de) \\ Jena University Hospital https://orcid.org/0000-0001-9163-5682
}

\section{Research}

Keywords: Acute respiratory infection, virus, rhinovirus, influenza virus, natural products, over-the-counter medication, cytotoxicity, antiviral activity

Posted Date: February 13th, 2020

DOI: https://doi.org/10.21203/rs.2.23461/v1

License: (c) (i) This work is licensed under a Creative Commons Attribution 4.0 International License. Read Full License 


\section{Abstract}

Background: Rhinoviruses and influenza viruses cause the majority of acute respiratory infections (ARIs). Symptoms of ARIs are commonly treated with over-the-counter products like ambroxol, bromhexine, and $\mathrm{N}$-acetylcysteine, as well as thyme and pelargonium extracts. Because the antiviral activity of these over-the-counter products has not been studied in a systematic way, the current study aimed to compare their antiviral effect against two rhinovirus and two influenza virus strains in an in vitro setting.

Methods: The cytotoxicity of ambroxol, bromhexine, and N-acetylcysteine, as well as of thyme and pelargonium extracts was analyzed in MadinDarby canine kidney (MDCK) and HeLa cells. The antiviral effect of these over-the-counter products was compared by analyzing the dosedependent inhibition (i) of rhinovirus A2- and B14-induced cytopathic effect in HeLa cells and (ii) of influenza virus A/Hong Kong/68 (subtype H3N2)- and A/Jena/8178/09 (subtype H1N1, pandemic)-induced cytopathic effect in MDCK cells at non-cytotoxic concentrations.

Results: The results revealed a good compatibility (no or only marginal cytotoxicity in MDCK and HeLa cells) of the agents that were tested. No antirhinoviral activity was detected at non-cytotoxic concentrations in this in vitro study setting. Ambroxol, bromhexine, and $\mathrm{N}$-acetylcysteine inhibited the influenza virus-induced cytopathic effect in MDCK cells no or less than $50 \%$. In contrast, a dose-dependent anti-influenza virus activity of thyme and pelargonium extracts was demonstrated.

Conclusions: The results of our comparative in vitro study indicate differences in the antiviral efficacy of ambroxol, bromhexine, and Nacetylcysteine, as well as of thyme and pelargonium extracts and suggest that the inhibition of influenza virus replication by thyme and pelargonium extracts may contribute to the beneficial effects of these plant extracts on ARI symptoms.

\section{Background}

Acute respiratory infections (ARIs) affect the upper and/or lower respiratory tract and account for the majority of infectious diseases worldwide. ARIs have a huge medical and economic impact through increased use of healthcare resources and loss in productivity. Severe infections of the lower respiratory tract even represent the fourth most common reason of death worldwide [1].

ARI are commonly caused by viruses [2]. In particular rhino- and influenza viruses are frequently diagnosed in ARIs with mild as well as severe respiratory symptoms, including acute lung injury $[3,4]$. Annual influenza epidemics are estimated to result in about 3 to 5 million cases of severe illness, and about 290000 to 650000 respiratory deaths [5]. Unfortunately, measures of prevention and treatment of rhinovirus infections do not exist, albeit the viral capsid, the protease, and polymerase have been identified as valid targets of inhibitors acting against a broad spectrum of rhinovirus serotypes [6, 7]. Even though influenza vaccines do exist, they are suboptimal applied [8-11]. The arsenal of drugs for treatment of influenza is limited and currently includes M2 ion channel blockers (amantadine and rimantadine), neuraminidase inhibitors (oseltamivir, zanamivir, laninamivir, and peramivir), and polymerase inhibitors (favipiravir and baloxavir) [12, 13]. Moreover, there is a permanent risk of emergence of drugresistant influenza viruses due to the high genetic variability based on point mutations and gene reassortment [14]. For example, the two M2 ion channel blockers are not recommended for use in monotherapy today because the circulating influenza A viruses are resistant [5]. Of note, ion channel blockers do not generally act against influenza B viruses due to structural differences in the viral target [15]. According to World Health Organization (WHO) recommendations, "patients with severe or progressive clinical illness associated with suspected or confirmed influenza virus infection (i.e. clinical syndromes of pneumonia, sepsis or exacerbation of chronic underling diseases) should be treated with antiviral drugs as soon as possible" [5]. Ideally treatment should be started within 48 hours following symptom onset to maximize therapeutic benefits, but drug administration should also be considered in patients presenting later in the course of illness [5].

The WHO further recommends that influenza patients who are not in a high-risk group "should be managed with symptomatic treatment and are advised, if symptomatic, to stay home in order to minimize the risk of infecting others in the community. Treatment focuses on relieving symptoms of influenza such as fever" [5]. In fact, the management of upper and lower respiratory tract infections often relies on the use of self-medicated overthe-counter (OTC) medicines [16].

According to a European study analyzing medication use in primary care patients with lower respiratory tract infections, $55.4 \%$ of patients selfmedicated before consultation and $21.5 \%$ after consultation. Mucolytic agents were amongst the most frequently used self-medications [16]. Mucolytic agents like ambroxol (a metabolite of bromhexine), bromhexine, and $\mathrm{N}$-acetylcysteine are also widely used in the therapy of chronic obstructive pulmonary disease (reduction of frequency and duration of exacerbations), bronchitis and sinusitis [17]. The anti-inflammatory and antioxidant properties of mucolytic agents have been shown to contribute to symptom relief $[17,18]$ but further research is needed to prove their efficacy $[16,17]$. Furthermore, thyme and pelargonium extracts are frequently used for symptomatic treatment of ARIs [19-21].

The antiviral potential of OTC medicines has been studied sporadically. Few studies were performed with ambroxol [22, 23], N-acetylcysteine [2427], and pelargonium extracts [28-30] in vitro and/or in vivo. The results are hard to compare because, in part, very high agent concentrations, different experimental conditions (e.g. cell lines or primary cells, different treatment schedules, infections dose, and controls), and study parameters (e.g. lethality and time of symptom release) were applied. Albeit beneficial treatment effects were shown in vitro and in vivo, it is difficult to draw conclusions on the effects of most of these self-medications on rhinovirus and/or anti-influenza virus replication. Although in vitro experiments 
cannot mimic the multifactorial and very complex in vivo situation and have only a limited predictive value for the clinical setting, antiviral effects shown in vitro can help to explain treatment success seen in vivo or in a clinical setting.

In this study, we aimed to comparatively analyze the effect of ambroxol, bromhexine, N-acetylcysteine, thyme, and pelargonium extract on the replication of rhinoviruses and influenza A viruses at non-cytotoxic micromolar concentrations in vitro. The well-known inhibitors pleconaril and zanamivir were used to validate the antiviral assays with rhinoviruses and influenza A viruses, respectively. To ensure the selectivity of the antiviral effect, the cytotoxicity of all extracts and compounds was analyzed in the cell lines used for antiviral studies.

\section{Methods}

\section{Reference compounds and test items}

As there are no drugs to treat rhinovirus infections, pleconaril, an inhibitor with well-known anti-rhinoviral activity was used to validate the antiviral assays. The sensitivity of rhinoviruses to pleconaril has been determined in previous studies [31, 32]. For influenza A viruses, the drug zanamivir was used as reference compound. The zanamivir sensitivity of the used influenza A viruses was known [33, 34]. Stock solutions of pleconaril and zanamivir (10 $000 \mu \mathrm{M})$ were prepared in dimethyl sulfoxide and bi-distilled water, respectively.

The cytotoxic and antiviral activities of ambroxol and bromhexine hydrochloride (Boehringer Ingelheim Pharma GmbH \& Co KG, Ingelheim, Germany), N-acetylcysteine (SIGMA-Aldrich Chemie GmbH, Schnelldorf, Germany), fluid thyme extract (R\&R Extrakte GmbH; Cologne, Germany), pelargonium extract (A. Nattermann \& Cie. GmbH; Cologne, Germany) were compared in this study. The thyme extract was used as provided. Stock solutions of the other test items were prepared in dimethyl sulfoxide in the following concentrations: $10000 \mu \mathrm{g} / \mathrm{ml}$ of pelargonium extract or 10000 $\mu \mathrm{M}$ for ambroxol, bromhexine, and N-acetylcysteine.

Working solutions of the reference compounds and test items were done in the test medium described in the following paragraph.

\section{Cell lines and virus strains}

HeLa Ohio (human cervix carcinoma) and Madin-Darby canine kidney (MDCK) cells allow the determination of cytotoxicity as well as antiviral activity of inhibitors against rhinoviruses and influenza A viruses, respectively. Cell culture growth medium for HeLa cells contained Eagle's minimal essential medium (EMEM), supplemented with 5\% newborn calf serum (NCS), 2 mM L-glutamine, and 1\% non-essential amino acids (NEAA). For MDCK cells, EMEM with 10\% NCS, 2 mM L-glutamine, and 1\% NEAA was used. The EMEM for anti-rhinoviral tests (test medium) in HeLa cells was supplemented with $2 \%$ NCS only. The antiviral tests with influenza A viruses were performed in MDCK cells with EMEM supplemented with $2.3 \%$ sodium bicarbonate, $2 \mu \mathrm{g} / \mathrm{ml}$ trypsin, $2 \mathrm{mM}$ L-glutamine, and 1\% NEAA (test medium).

Viruses included in this study were rhinovirus A2 (Institute of Biochemistry, University, Vienna, AUT), rhinovirus B14 (Charité, Berlin, Germany), the H3N2 influenza virus A/Hong Kong/68 (Schaper and Brümmer, Salzgitter, Germany), and the A(H1N1)pdm09 influenza virus A/Jena/8178/09 (isolated and kindly provided by Andy Krumbholz (34)). Rhinoviruses and influenza A viruses were grown and titrated in HeLa and MDCK cells, respectively. The determined virus titers of rhinovirus $A 2$, rhinovirus $B 14$, influenza virus $A / H o n g ~ K o n g / 68$, and influenza virus $A / J e n a / 8178 / 09$ were $6.3 \times 10^{6} \mathrm{TCID}_{50} / \mathrm{ml}, 2.0 \times 10^{6} \mathrm{TCID}_{50} / \mathrm{ml}, 2.0 \times 10^{7} \mathrm{TCID} 50 / \mathrm{ml}$, and $6.3 \times 10^{7} \mathrm{TCID}_{50} / \mathrm{ml}$, respectively. Aliquots of the virus working passages were stored at $-80^{\circ} \mathrm{C}$ until use.

\section{Cytotoxicity determination}

HeLa Ohio and MDCK cells were seeded at $1.6 \times 10^{4}$ and $2.3 \times 10^{4}$, cells/well in $100 \mu$ l growth medium in 96 well flat-bottomed microtiter plates, respectively. The cytotoxicity of the test compounds was determined on two-day-old confluent cell monolayers grown in the internal 60 wells of a microtiter plate $\left(5 \%\right.$ carbon dioxide, $\left.37^{\circ} \mathrm{C}\right)$. After removal of the growth medium, $50 \mu \mathrm{l}$ of test medium and the eight half-log dilutions of the reference compounds pleconaril or zanamivir, or eight half-log dilutions of test items in test medium (each concentration in duplicates) were added. The concentration range of reference compounds applied to cytotoxicity assays was $0.0316-100 \mu \mathrm{M}$. The concentration range of test items was $0.00033-0.5 \% \mathrm{v} / \mathrm{v}$ for thyme extract, $0.0316-100 \mu \mathrm{g} / \mathrm{ml}$ for pelargonium extract, and 0.0316-100 $\mu \mathrm{M}$ for ambroxol, bromhexine, and $\mathrm{N}$-acetylcysteine. Six cell control wells were incubated with $100 \mu \mathrm{l}$ test medium. After 72 hours of incubation at $37^{\circ} \mathrm{C}$ in $5 \%$ carbon dioxide atmosphere, the Dynex Immuno Assay System (Guernsey, UK), developed for automated ELISA techniques, was applied to gently wash, stain, measure, and analyze the viability of the cell monolayers [35]. Cell viability was evaluated as the percentage of the mean value of optical density resulting from the 6 cell controls, which was set $100 \%$. The inhibitor concentration that reduces the viability of the treated cells in comparison to untreated control cells (no inhibitor) by half is called $50 \%$ cytotoxic concentration $\left(\mathrm{CC}_{50}\right)$. A minimum of three independent experiments were performed.

\section{Antiviral assays}

Page $3 / 10$ 
Rhinoviruses and influenza viruses cause a cytopathic effect (CPE) in HeLa and MDCK cells, respectively. The CPE can be quantified after staining the cells with crystal violet and dye elution by optical density measurement as described before [32, 35]. The ability of certain compounds to protect cells from CPE can be analyzed using CPE inhibitory assays. Seeding and growth of MDCK cells with influenza viruses for CPE inhibitory assays was done similarly to the cytotoxicity assay. HeLa cells were incubated for one day only before adding rhinoviruses in anti-rhinovirus assays. After the aspiration of the cell growth medium, $50 \mu$ of test medium (mock-treatment of cell and virus controls) or 8 half-log dilutions of reference compounds or test items in test medium, and a certain multiplicity of infection (MOI) of the test virus in a volume of $50 \mu \mathrm{l}$ of the test medium, were added to the cell monolayers. The MOI of RV-A2, RV-B14, HK/68, and Jena/8178 was adjusted to $0.03,0.01,0.008$, and 0.005 TCID ${ }_{50} /$ cell, respectively. The concentration range of reference compounds (pleconaril or zanamivir) applied to the CPE inhibitory assays was $0.0003-1 \mu \mathrm{M}$. The concentration range of test items applied to test was $0.00033-0.5 \% \mathrm{v} / \mathrm{v}$ for thyme extract, $0.0316-100 \mu \mathrm{g} / \mathrm{ml}$ for pelargonium extract, and 0.0316 $100 \mu \mathrm{M}$ for ambroxol, bromhexine, and N-acetylcysteine. Six wells of non-infected and six wells of infected cells (both mock-treated with test medium) served as cell and virus control, respectively, on each plate.

Plates were incubated at $37^{\circ} \mathrm{C}$ in a humidified atmosphere with $5 \% \mathrm{CO}_{2}$ for 48 hours (HK/68 and Jena/8178) or 72 hours (RV-A2), or at $33{ }^{\circ} \mathrm{C}$ for 72 hours (RV-B14). Thereafter, the cell monolayers were fixed and stained with a $0.03 \%$ crystal violet solution in $2 \%$ ethanol and $3 \%$ formalin in water with the Dynex Immuno Assay System (Guernsey, UK) following the procedure described elsewhere [35]. The percentage of antiviral activities of the test compounds were calculated according to Pauwels et al. [36] using the following equation: antiviral activity $=[$ (mean optical density of 6 cell controls - mean optical density of 6 virus controls)/(optical density of test - mean optical density of 6 virus controls)] $\times 100 \%$. A 100\% CPE inhibition means that $100 \%$ of virus-infected, inhibitor-treated cells were viable. At least three independent experiments were performed.

\section{Statistical methods}

The $50 \%$ cytotoxic and inhibitory concentrations $\left(\mathrm{CC}_{50}\right.$ and $\mathrm{IC}_{50}$ values) were calculated from dose-response curves. Linear regression analysis using Microsoft Excel 2010 was applied in the linear scaled dose-dependent sample concentrations. Means and standard deviations (SD) of the $\mathrm{CC}_{50}$ and $\mathrm{IC}_{50}$ were calculated using Microsoft Excel 2010.

\section{Results}

\section{Cytotoxicity in HeLa cells and anti-rhinovirus activity}

The cytotoxicity of test items for HeLa cells was quantified using a crystal violet staining procedure at day three after compound addition. The results are summarized in Table 1 and Fig. 1 . Only non-cytotoxic concentrations ( ${ }^{3} 90 \%$ viability as compared to the mean optical density value of six cell control wells which was set at $100 \%$ cell viability) were used to assess antiviral activity to avoid an impairment of virus growth due to cytotoxic effects of the test items.

The control compound pleconaril exerted a marked dose-dependent cytotoxic effect in HeLa cells when applied at concentrations higher than $10 \mu \mathrm{M}$ (Fig. 1a). However, pleconaril dose-dependently inhibited the rhinovirus A2- and rhinovirus B14-induced CPE at low micromolar concentrations that were well tolerated by HeLa cells (Fig. 1a, Table 1). Thus, the $\mathrm{CC}_{50} / \mathrm{IC}_{50}$ ratio indicating the selectivity of pleconaril activity was $>3500$.

With the exception of thyme extract, all test items were well tolerated in Hela cells. N-acetylcysteine was non-cytotoxic in the tested concentration range (Table 1). However, in contrast to pleconaril and as seen in Fig. 1b-1f, none of the tested OTCs reduced the rhinovirus A2- and rhinovirus B14induced CPE at concentrations that are non-cytotoxic for HeLa cells. A slight inhibition of rhinovirus-induced CPE of was seen at concentrations affecting also cell viability.

\section{Cytotoxicity in MDCK cells and anti-influenza A virus activity}

The control compound zanamivirwas well tolerated by MDCK cells (Fig. 2a). Zanamivir was highly active against both Influenza A viruses, and inhibited the influenza virus A/Hong Kong/68- and influenza virus A/Jena/8178/09-induced CPE at nano- and micromolar concentrations in a dosedependent manner (Fig. 2a, Table 1).

As seen in Table 1, Fig. 2b and 2d, N-acetylcysteine and ambroxol did not show any effect on influenza virus A/Hong Kong/68 and influenza virus A/Jena/8178/09 at non-cytotoxic concentrations (MDCK cell viability $>90 \%$ ). The induction of CPE was inhibited by approximately $50 \%$ at a concentration of 31.6 and/or $100 \mu \mathrm{M}$. At this concentration, an unspecific inhibition of viral replication mediated by the measured cytotoxic effect cannot be excluded.

Bromhexine was cytotoxic at $100 \mu \mathrm{M}$ (Fig. 2c). At $31.6 \mu \mathrm{M}$, the influenza virus A/Hong Kong/68-induced CPE was reduced by $30 \%$. Thyme extract was cytotoxic at the maximum tested concentration of $0.5 \% \mathrm{v} / \mathrm{v}$ (Fig. 2e). At lower, non-cytotoxic concentrations, the thyme extract dosedependently decreased the influenza virus A/Hong Kong/68- and influenza virus A/Jena/8178/09-induced CPE indicating an antiviral activity. The

Page $4 / 10$ 
pelargonium extract exerted the strongest dose-dependent anti-influenza virus effect of all tested agents. It showed IC ${ }_{50}$ values of approximately 10 $\mu \mathrm{M}$ against both influenza virus A/Hong Kong/68- and influenza virus A/Jena/8178/09 (Table 1, Fig. 2f). Moreover, pelargonium extract was well tolerated in MDCK cells.

In summary, all test items were well tolerated in confluent HeLa and MDCK cells. However, there was no antiviral activity against rhinovirus A2 and rhinovirus B14 at non-cytotoxic concentrations. Thyme and pelargonium extract inhibited the influenza virus-induced cytopathic effect in MDCK cells, whereas the other test items exerted a small effect or no effect at all.

\section{Discussion}

In the present study, the effect of five commonly used OTCs for treatment of ARIs on the replication of two rhinoviruses and two influenza A viruses was compared under standardized experimental conditions (e.g. MOI, compound treatment, readout, control compounds) in an in vitro setting. The previously reported antiviral activities of the control compounds pleconaril [31, 32] and zanamivir [33, 34] were fully confirmed here.

The results indicate a different anti-rhinovirus and anti-influenza A virus potential of ambroxol, bromhexine, $\mathrm{N}$-acetylcysteine, thyme and pelargonium extracts. At non-cytotoxic concentrations, neither bromhexine nor its metabolite ambroxol $(0.0316-100 \mu \mathrm{M})$ exerted a $\geq 50 \% \mathrm{CPE}$ inhibition against the tested rhinoviruses and influenza A viruses in HeLa and MDCK cells, respectively, when added immediately before virus challenge to the cells. Ambroxol reaches a mean peak human plasma concentration of about $0.2 \mu \mathrm{M}$ [37], a concentration that was non-cytotoxic and did not act antiviral here. In contrast, a 3-day pretreatment of primary cultures of human tracheal epithelial cells with ambroxol at a concentration of at least $0.1 \mu \mathrm{M}$ reduced the viral titer of RV-B14 in the supernatant in a concentration-dependent manner [22]. According to Yamaya et al. [22], a 3-day pretreatment of the cells with ambroxol indirectly affected the RV-B14 infection by reducing the virus receptor expression, the number of acidic endosomes, and the fluorescence intensity of the acidic endosomes compared to untreated or vehicle-treated cells. The antiviral effect of ambroxol added immediately before virus challenge was not studied by these authors. Therefore, it remains unclear whether the different study outcome is based on the different cells used, the pretreatment with ambroxol or both. To the best of our knowledge, there are no publications on the anti-influenza virus effect of ambroxol in vitro. In mouse airways, influenza virus replication was indirectly suppressed by ambroxol-induced increase of the concentration of suppressors of viral replication, such as pulmonary surfactant, mucus protease inhibitor, and immunoglobulin A [23]. The absence of pulmonary surfactant, mucus protease inhibitor, and immunoglobulin A in vitro could explain the lack of anti-viral activity in in vitro experiments.

No antiviral activity was found for the antioxidant molecule $\mathrm{N}$-acetylcysteine $(0.0316-100 \mu \mathrm{M})$ in the present study. There are no publications on the anti-rhinovirus effect of $\mathrm{N}$-acetylcysteine in vitro. The negative results obtained for influenza A viruses seem to be in contradiction with previous findings obtained with influenza A viruses in A549 cells $[25,26]$. However, this previously published anti-influenza virus activity was observed after treating A549 cells at much higher concentrations (10 and/or $15 \mathrm{mM}$ ), starting with a 1-hour or 24-hour pre-incubation period prior to virus infection. In striking contrast, the maximal plasma concentration following the application of $600 \mathrm{mg}$ of $\mathrm{N}$-acetylcysteine is only about $16 \mu \mathrm{M}$ [38]. There are two reports on the protective effects (reduction of lethality and/or body weight loss) of $\mathrm{N}$-acetylcysteine ( $0.2 \mathrm{or} 1 \mathrm{~g} / \mathrm{kg}$ body weight per day) in models of influenza infection in mice [27,39]. Both reports explain the observed protective effects by the antioxidant activity of N-acetylcysteine attenuating pulmonary inflammation which does not play a role in in vivo experiments. A third report did not confirm the protective effect of $\mathrm{N}$ acetylcysteine ( $1 \mathrm{~g} / \mathrm{kg}$ body weight per day) on survival and the mean survival time in influenza A virus-infected mice [24].

The thyme extract was well tolerated in both HeLa and MDCK cells. It was inactive against rhinoviruses, but reduced the influenza A virus-induced cytopathic effect at non-cytotoxic dilutions $(0.03-0.33 \% \mathrm{v} / \mathrm{v})$ in a dose-dependent manner. Thyme extract is also known to inhibit herpes simplex virus infections in vitro [40,41] by inactivating the infectivity of the virus [41].

Pelargonium extract did not inhibit the induction of CPE by rhinoviruses A2 and B14 in HeLa cells. However, it was shown to reduce rhinovirus A16 infection of human primary bronchial epithelial cells by down-regulating cell membrane docking proteins and up-regulating host defense proteins, as described recently [29]. Pelargonium extract exerted the best anti-influenza A virus activity with mean $\mathrm{IC}_{50}$ values of 7.80 and $11.67 \mu \mathrm{g} / \mathrm{ml}$ for the two influenza A virus strains tested (H1N1: Jena/8178 and H3N2: HK/69). These results assort well with data published for Pelargonium sidoides extract EPs ${ }^{\circledR} 7630$ and other influenza A virus strains of subtype H1N1 and H3N2 in MDCK cells elsewhere [28, 30]. EPs ${ }^{\circledR} 7630$ impaired viral hemagglutination as well as neuraminidase activity and inhibited viral replication when added to cells two hours before or at the time of virus challenge [30]. Polyphenolic compounds, in particular oligomeric and polymeric proanthocyanidins based on gallocatechin and epigallocatechin moieties (prodelphinidins), represent the antiviral active compounds in EPs $\AA 7630$. The benefit of EPs ${ }^{\circledR} 7630$ treatment was also demonstrated in a mouse model of influenza where the extract was administered by inhalation [30].

\section{Conclusions}

The results obtained in the present in vitro study suggest that the inhibition of influenza virus replication by thyme and pelargonium extract may contribute to the beneficial effects of these plant extracts on ARI symptoms. 


\section{Abbreviations}

ARIs: acute respiratory infections; $\mathrm{CC}_{50}: 50 \%$ cytotoxic concentration; CPE: cytopathic effect; EMEM: Eagle's minimal essential medium; IC ${ }_{50}$ : 50\% inhibitory concentration; MDCK: Madin-Darby canine kidney; MOI: multiplicity of infection; NCS: newborn calf serum; NEAA: non-essential amino acids; OTC: over-the-counter; SD: standard deviation; WHO: world health organization.

\section{Declarations}

Ethics approval and consent to participate: Not applicable.

Consent for publication: Not applicable.

Availability of data and materials: The datasets used and/or analyzed during the current study are available from the corresponding author on reasonable request.

Competing interests: The authors declare that they have no competing interest.

Funding: This work was financially supported by Sanofi-Aventis Deutschland GmbH. No influence was taken on study design, analysis and interpretation of data.

Authors' contributions: CW analyzed the cytotoxicity and anti-rhinoviral activity of the test items in HeLa cells. MS designed the study and analyzed the cytotoxicity and anti-influenza virus activity of the test items in MDCK cells and wrote the manuscript. Both authors read and approved the final manuscript.

Acknowledgements: Not applicable.

\section{References}

1. WHO. Global health estimates 2016: Deaths by Cause, Age, Sex, by Country and by Region, 2000-2016 2018. Available from: http://fmrglobalhealth.com/frame/top10.html.

2. Gonzales R, Sande MA. Uncomplicated acute bronchitis. Annals of internal medicine. 2000;133(12):981-91.

3. Heikkinen T, Jarvinen A. The common cold. Lancet. 2003;361(9351):51-9.

4. Taylor S, Lopez P, Weckx L, Borja-Tabora C, Ulloa-Gutierrez R, Lazcano-Ponce E, et al. Respiratory viruses and influenza-like illness: Epidemiology and outcomes in children aged 6 months to 10 years in a multi-country population sample. The Journal of infection. 2017;74(1):29-41.

5. WHO. Fact sheet on seasonal influenza. 2018. Available from: https://www.who.int/en/news-room/fact-sheets/detail/influenza-(seasonal).

6. Egorova A, Ekins S, Schmidtke M, Makarov V. Back to the future: Advances in development of broad-spectrum capsid-binding inhibitors of enteroviruses. European journal of medicinal chemistry. 2019;178:606-22.

7. Rollinger JM, Schmidtke M. The human rhinovirus: human-pathological impact, mechanisms of antirhinoviral agents, and strategies for their discovery. Medicinal research reviews. 2011;31(1):42-92.

8. Cataldi JR, O'Leary ST, Lindley MC, Hurley LP, Allison MA, Brtnikova M, et al. Survey of Adult Influenza Vaccination Practices and Perspectives Among US Primary Care Providers (2016-2017 Influenza Season). Journal of general internal medicine. 2019.

9. Hagel S, Ludewig K, Moeser A, Baier M, Loffler B, Schleenvoigt B, et al. Characteristics and management of patients with influenza in a German hospital during the 2014/2015 influenza season. Infection. 2016;44(5):667-72.

10. Jorgensen P, Mereckiene J, Cotter S, Johansen K, Tsolova S, Brown C. How close are countries of the WHO European Region to achieving the goal of vaccinating $75 \%$ of key risk groups against influenza? Results from national surveys on seasonal influenza vaccination programmes, 2008/2009 to 2014/2015. Vaccine. 2018;36(4):442-52.

11. Wang Q, Yue N, Zheng M, Wang D, Duan C, Yu X, et al. Influenza vaccination coverage of population and the factors influencing influenza vaccination in mainland China: A meta-analysis. Vaccine. 2018;36(48):7262-9.

12. Mifsud EJ, Hayden FG, Hurt AC. Antivirals targeting the polymerase complex of influenza viruses. Antiviral research. 2019;169:104545.

13. Naesens L, Stevaert A, Vanderlinden E. Antiviral therapies on the horizon for influenza. Current opinion in pharmacology. 2016;30:106-15.

14. Johnson KEE, Song T, Greenbaum B, Ghedin E. Getting the flu: 5 key facts about influenza virus evolution. PLoS pathogens. 2017;13(8):e1006450.

15. To J, Torres J. Viroporins in the Influenza Virus. Cells. 2019;8(7).

16. Hamoen M, Broekhuizen BD, Little P, Melbye H, Coenen S, Goossens H, et al. Medication use in European primary care patients with lower respiratory tract infection: an observational study. The British journal of general practice : the journal of the Royal College of General 
Practitioners. 2014;64(619):e81-91.

17. Scaglione F, Petrini O. Mucoactive Agents in the Therapy of Upper Respiratory Airways Infections: Fair to Describe Them Just as Mucoactive? Clinical medicine insights Ear, nose and throat. 2019;12:1179550618821930.

18. Paleari D, Rossi GA, Nicolini G, Olivieri D. Ambroxol: a multifaceted molecule with additional therapeutic potentials in respiratory disorders of childhood. Expert opinion on drug discovery. 2011;6(11):1203-14.

19. Agbabiaka TB, Guo R, Ernst E. Pelargonium sidoides for acute bronchitis: a systematic review and meta-analysis. Phytomedicine : international journal of phytotherapy and phytopharmacology. 2008;15(5):378-85.

20. Careddu D, Pettenazzo A. Pelargonium sidoides extract EPs 7630: a review of its clinical efficacy and safety for treating acute respiratory tract infections in children. International journal of general medicine. 2018;11:91-8.

21. Salehi B, Mishra AP, Shukla I, Sharifi-Rad M, Contreras MDM, Segura-Carretero A, et al. Thymol, thyme, and other plant sources: Health and potential uses. Phytotherapy research : PTR. 2018;32(9):1688-706.

22. Yamaya M, Nishimura H, Nadine LK, Ota C, Kubo H, Nagatomi R. Ambroxol inhibits rhinovirus infection in primary cultures of human tracheal epithelial cells. Archives of pharmacal research. 2014;37(4):520-9.

23. Yang B, Yao DF, Ohuchi M, Ide M, Yano M, Okumura Y, et al. Ambroxol suppresses influenza-virus proliferation in the mouse airway by increasing antiviral factor levels. The European respiratory journal. 2002;19(5):952-8.

24. Garozzo A, Tempera G, Ungheri D, Timpanaro R, Castro A. N-acetylcysteine synergizes with oseltamivir in protecting mice from lethal influenza infection. International journal of immunopathology and pharmacology. 2007;20(2):349-54.

25. Geiler J, Michaelis M, Naczk P, Leutz A, Langer K, Doerr HW, et al. N-acetyl-L-cysteine (NAC) inhibits virus replication and expression of proinflammatory molecules in A549 cells infected with highly pathogenic H5N1 influenza A virus. Biochemical pharmacology. 2010;79(3):413-20.

26. Mata M, Morcillo E, Gimeno C, Cortijo J. N-acetyl-L-cysteine (NAC) inhibit mucin synthesis and pro-inflammatory mediators in alveolar type II epithelial cells infected with influenza virus A and B and with respiratory syncytial virus (RSV). Biochemical pharmacology. 2011;82(5):548-55.

27. Ungheri D, Pisani C, Sanson G, Bertani A, Schioppacassi G, Delgado R, et al. Protective effect of n-acetylcysteine in a model of influenza infection in mice. International journal of immunopathology and pharmacology. 2000;13(3):123-8.

28. Michaelis M, Doerr HW, Cinatl J, Jr. Investigation of the influence of EPs(R) 7630, a herbal drug preparation from Pelargonium sidoides, on replication of a broad panel of respiratory viruses. Phytomedicine : international journal of phytotherapy and phytopharmacology. 2011;18(5):384-6.

29. Roth M, Fang L, Stolz D, Tamm M. Pelargonium sidoides radix extract EPs 7630 reduces rhinovirus infection through modulation of viral binding proteins on human bronchial epithelial cells. PloS one. 2019;14(2):e0210702.

30. Theisen LL, Muller CP. EPs(R) 7630 (Umckaloabo(R)), an extract from Pelargonium sidoides roots, exerts anti-influenza virus activity in vitro and in vivo. Antiviral research. 2012;94(2):147-56.

31. Ledford RM, Patel NR, Demenczuk TM, Watanyar A, Herbertz T, Collett MS, et al. VP1 sequencing of all human rhinovirus serotypes: insights into genus phylogeny and susceptibility to antiviral capsid-binding compounds. Journal of virology. 2004;78(7):3663-74.

32. Makarov VA, Braun H, Richter M, Riabova OB, Kirchmair J, Kazakova ES, et al. Pyrazolopyrimidines: Potent Inhibitors Targeting the Capsid of Rhino- and Enteroviruses. ChemMedChem. 2015;10(10):1629-34.

33. Schade D, Kotthaus J, Riebling L, Kotthaus J, Muller-Fielitz H, Raasch W, et al. Zanamivir Amidoxime- and N-Hydroxyguanidine-Based Prodrug Approaches to Tackle Poor Oral Bioavailability. Journal of pharmaceutical sciences. 2015;104(9):3208-19.

34. Walther E, Xu Z, Richter M, Kirchmair J, Grienke U, Rollinger JM, et al. Dual Acting Neuraminidase Inhibitors Open New Opportunities to Disrupt the Lethal Synergism between Streptococcus pneumoniae and Influenza Virus. Frontiers in microbiology. 2016;7:357.

35. Schmidtke M, Schnittler U, Jahn B, Dahse H, Stelzner A. A rapid assay for evaluation of antiviral activity against coxsackie virus B3, influenza virus $A$, and herpes simplex virus type 1. Journal of virological methods. 2001;95(1-2):133-43.

36. Pauwels R, Balzarini J, Baba M, Snoeck R, Schols D, Herdewijn P, et al. Rapid and automated tetrazolium-based colorimetric assay for the detection of anti-HIV compounds. Journal of virological methods. 1988;20(4):309-21.

37. Su F, Wang F, Gao W, Li H. Determination of ambroxol in human plasma by high performance liquid chromatography-electrospray ionization mass spectrometry (HPLC-MS/ESI). Journal of chromatography B, Analytical technologies in the biomedical and life sciences. 2007;853(12):364-8.

38. Borgstrom L, Kagedal B. Dose dependent pharmacokinetics of N-acetylcysteine after oral dosing to man. Biopharmaceutics \& drug disposition. 1990;11(2):131-6.

39. Zhang RH, Li CH, Wang CL, Xu MJ, Xu T, Wei D, et al. N-acetyl---cystine (NAC) protects against H9N2 swine influenza virus-induced acute lung injury. International immunopharmacology. 2014;22(1):1-8.

40. Rajbhandari M, Mentel R, Jha PK, Chaudhary RP, Bhattarai S, Gewali MB, et al. Antiviral activity of some plants used in Nepalese traditional medicine. Evidence-based complementary and alternative medicine : eCAM. 2009;6(4):517-22. 
41. Toujani MM, Ritta M, Civra A, Genovese S, Epifano F, Ghram A, et al. Inhibition of HSV-2 infection by pure compounds from Thymus capitatus extract in vitro. Phytotherapy research : PTR. 2018;32(8):1555-63.

\section{Table}

Table 1 Cytotoxicity and antiviral activity of test items and control compounds

\begin{tabular}{|c|c|c|c|c|c|c|c|c|c|c|c|c|c|c|c|}
\hline \multirow[t]{4}{*}{$\begin{array}{l}\text { Compounds/ } \\
\text { Extracts }\end{array}$} & \multirow[t]{4}{*}{ units } & \multirow{2}{*}{\multicolumn{4}{|c|}{$\begin{array}{c}50 \% \text { cytotoxic } \\
\text { concentration in }\end{array}$}} & \multicolumn{8}{|c|}{$50 \%$ inhibitory concentration against } & \multirow{2}{*}{\multicolumn{2}{|c|}{$\begin{array}{c}\text { Selectivity index } \\
\text { against } \\
\text { influenza A viruses }\end{array}$}} \\
\hline & & & & & & \multicolumn{4}{|c|}{ Rhinoviruses } & \multicolumn{4}{|c|}{ influenza A viruses } & & \\
\hline & & \multicolumn{2}{|c|}{ HeLa cells } & \multicolumn{2}{|c|}{ MDCK cells } & \multicolumn{2}{|c|}{ A2 } & \multicolumn{2}{|c|}{ B14 } & \multicolumn{2}{|c|}{ Jena/8178 } & \multicolumn{2}{|c|}{$\mathrm{HK} / 68$} & Jena/8178 & HK/68 \\
\hline & & mean & SD & mean & SD & mean & SD & mean & SD & mean & SD & mean & SD & & \\
\hline Pleconaril & $\mu \mathrm{M}$ & 28.75 & 8.06 & n.d. & & 0.008 & 0.003 & 0.025 & 0.009 & n.d. & & n.d. & & & \\
\hline Zanamivir & $\mu \mathrm{M}$ & n.d. & & $>100 *$ & & n.d. & & n.d. & & 0.007 & 0.004 & 0.008 & 0.003 & $>14285$ & $>12500$ \\
\hline Bromhexine & $\mu \mathrm{M}$ & 51.85 & 9.80 & 51.29 & 9.08 & n.a. & & n.a. & & n.a. & & n.a. & & & \\
\hline Ambroxol & $\mu \mathrm{M}$ & 61.24 & 42.03 & $>100^{*}$ & & n.a. & & n.a. & & n.a. & & n.a. & & & \\
\hline $\begin{array}{l}\mathrm{N}- \\
\text { acetylcysteine }\end{array}$ & $\mu \mathrm{M}$ & $>100 *$ & & $>100 *$ & & n.a. & & n.a. & & n.a. & & n.a. & & & \\
\hline $\begin{array}{l}\text { Thyme } \\
\text { extract }\end{array}$ & $\% \mathrm{v} / \mathrm{v}$ & $>0.5^{*}$ & & 0.32 & 0.07 & n.a. & & n.a. & & 0.03 & 0.01 & 0.03 & 0.00 & 11 & 11 \\
\hline $\begin{array}{l}\text { Pelargonium } \\
\text { extract }\end{array}$ & $\mu \mathrm{g} / \mathrm{ml}$ & $>100 *$ & & $>100 *$ & & n.a. & & n.a. & & 7.80 & 2.55 & 11.67 & 6.03 & $>13$ & $>9$ \\
\hline
\end{tabular}

Means and standard deviation (SD) of the calculated 50\% cytotoxic and the 50\% inhibitory concentrations are shown.

n.d.: not determined; n.a.: not active up to the maximum non-cytotoxic concentration.

*More than $50 \%$ viability at the maximum tested concentration. Therefore, the $50 \%$ cytotoxic concentration is assumed to be higher than $100 \mu \mathrm{g} / \mathrm{ml}, 100 \mathrm{\mu M}$, $0.5 \% \mathrm{v} / \mathrm{v}$.

\section{Figures}



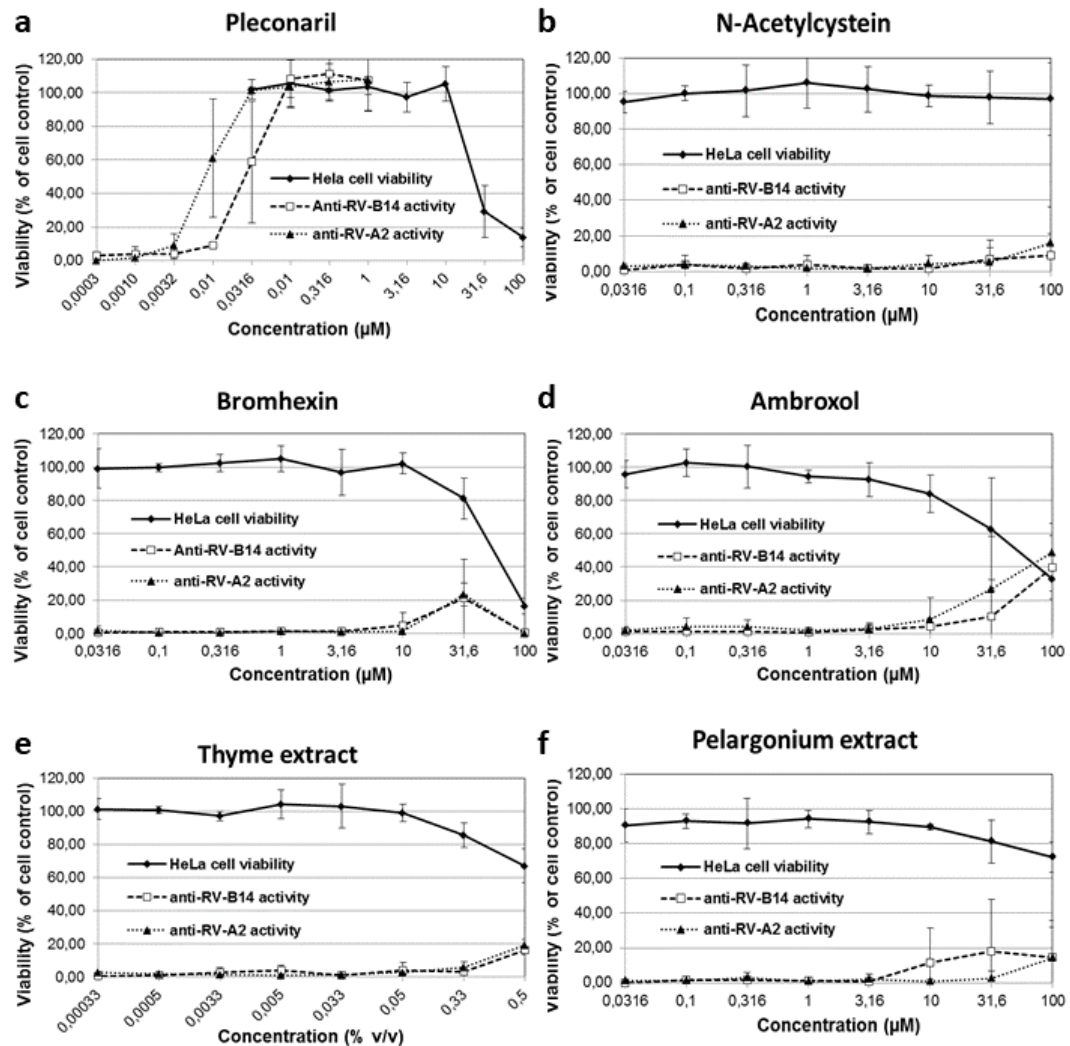

\section{Figure 1}

Dose-dependent cytotoxicity and anti-rhinovirus activity of pleconaril and test items in HeLa cells. The cytotoxicity and anti-rhinovirus activity of pleconaril (a), N-acetylcysteine (b), bromhexine (c), ambroxol (d), thyme extract (e), and pelargonium extract (f) were analyzed in HeLa cells.

Rhinovirus A2 (RV-A2) and anti-rhinovirus B14 (RV-B14) were included as test viruses in the antiviral studies. Means and standard deviations of at least three independent experiments are shown. 
a

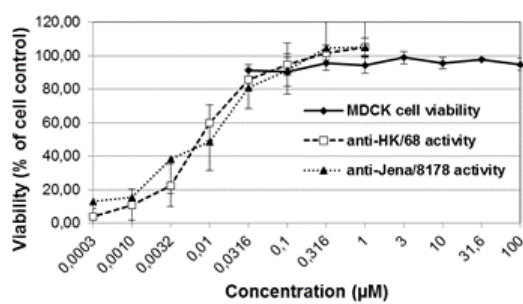

Concentration $(\mu \mathrm{M})$

C

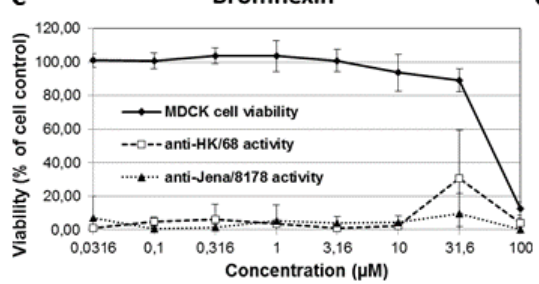

Concentration $(\mu \mathrm{M})$

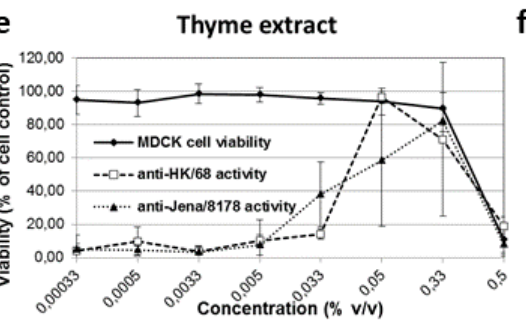

b

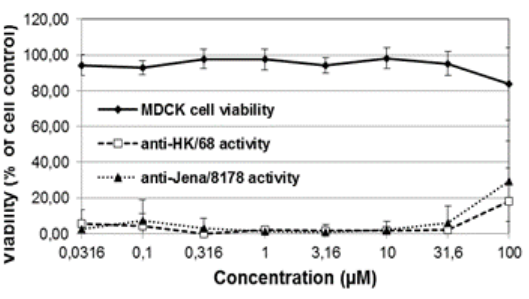

d

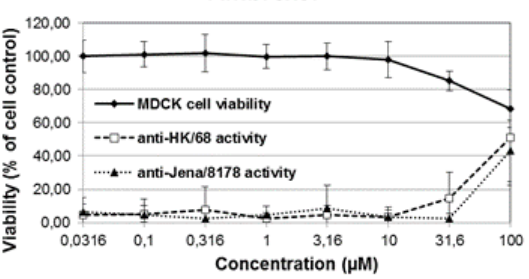

f

Pelargonium extract

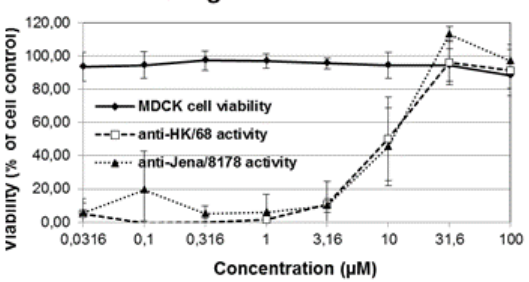

Figure 3

Dose-dependent cytotoxicity and anti-influenza virus activity of zanamivir and test items in MDCK cells. The cytotoxicity and anti-rhinovirus activity of pleconaril (a), N-acetylcysteine (b), bromhexine (c), ambroxol (d), thyme extract (e), and pelargonium extract (f) were analyzed in HeLa cells. Influenza virus A/Hong Kong/68 (HK/68) and influenza virus A/Jena/8178/09 (Jena/8178) were included in the antiviral studies. Means and standard deviations of at least three independent experiments are shown. 